\title{
Die Zeichnung als Befehl
}

\author{
Wolfgang Pircher, Wien
}

Um Wittgensteins Bildtheorie des Tractatus zu interpretieren, haben Kelly Hamilton und Michael Biggs ${ }^{1}$ Formen des technischen Zeichnens aufgerufen, die Wittgenstein während seiner Berliner Zeit an der TH Charlottenburg sehr wahrscheinlich vertraut waren. Auch Brian McGuiness geht darauf ein, wenn er sagt, daß ein Hauptgedanke des Tractatus, nämlich „,die Vorstellung des Satzes als Bild, in vielen auf Überlegungen zurück (geht), die mit den Lehrfächern von Professor Jolles zu tun haben“, ${ }^{2}$ also Darstellende Geometrie und Graphische Statik. Hamilton wiederum gibt den Hinweis, daß Wittgenstein Bilder in einer „projektiven Beziehung“ zur Realität gesehen habe. Eine solche Beziehung wiederum sei typisch für den Ingenieur und die Darstellende Geometrie. Biggs hingegen setzt weniger auf die Darstellende Geometrie als auf die Graphische Statik und dynamische Modelle. Man kann das zum Anlaß nehmen, um einen genaueren Blick auf diese beiden Lehrfächer zu werfen.

\section{Die Sprache des Ingenieurs}

Bei der Darstellenden Geometrie handelt es sich um eine zeichnerische Methode der Parallelprojektion, mittels welcher ein räumlicher Gegenstand, der streng definierbar sein muß, zweimal dargestellt wird, durch eine Seiten- und eine Aufsicht. Das geschieht durch Projektion auf zwei Ebenen, die senkrecht aufeinander stehend gedacht werden. Tatsächlich befinden sich beide in einer Fläche, was die Vorstellungskraft des Zeichners auf eine gewisse Probe stellt. Die Eigenart des abgebildeten Gegenstandes ist es nicht einfach gegeben zu sein, sondern konstruktiv aus den zeichnerischen Operationen hervorzuwachsen. Das ist der Grund, warum Gaspard Monge, der Erfinder der Darstellenden Geometrie, sagt, sie ermögliche Neues 
zu erfinden. Entstanden aus der Kunst des Steinschnittes, ist die Darstellende Geometrie für das moderne technische Zeichnen in einer Hinsicht wichtig: in der kontinentaleuropäischen Darstellungsweise wird ein technischer Gegenstand gemäß der Projektionsregel von Monge abgebildet, d. h. die rechte Seite wird links vom Seitenriss gezeichnet, die rechte entsprechend links und die Aufsicht wird unter den Seitenriss platziert. Die gesamte Darstellung befindet sich daher im ersten Quadranten, was elegant ist, weil alle Zahlenwerte positiv sind. Das geht aber mit der Schwierigkeit einher, die Projektion auf die Zeichenebene gleichsam durch den Gegenstand hindurch vorzunehmen, eine Leistung der Vorstellungskraft, die man gegen Ende des 19. Jahrhunderts den U.S.-amerikanischen Arbeitern nicht zutraute und dementsprechend die Zeichenpraxis änderte: die rechte Seite wird rechts gezeichnet, die linke links und die Aufsicht oberhalb des Seitenrisses platziert. ${ }^{3}$ Abgesehen davon ist die Darstellende Geometrie für das technische Zeichnen nicht maßgebend, sie spielt und spielte ihre Rolle vornehmlich in der Ingenieurspädagogik, wo sie nach den Worten von Monge an das genaue Zeichnen gewöhnen soll. Er nannte sie auch die dem Ingenieur unerlässliche Sprache. Das wird in der Folge oft wiederholt werden. Der Ingenieur spricht indem er zeichnet oder, im Stadium der sog. „sprechenden Skizze“, spricht er gleichzeitig mit dem Zeichnen (Skizzieren). Aber dieses Zeichnen ist kein Abzeichnen von Gegenständen, die bereits existieren, sondern ist Element eines Entwurfsprozesses, der schließlich in einen Konstruktionsprozess übergehen soll und dieser wiederum soll im Resultat der Werkstattzeichnungen gleichsam den Befehl des Ingenieurs repräsentieren, ein technisches Objekt exakt nach seinen Vorgaben zu bauen.

Über die Vermittlung von Jolles, der neben der Darstellenden Geometrie auch die Graphische Statik unterrichtete, könnte Wittgenstein mit dieser vertraut gemacht worden sein. Der damals prominenteste Vertreter dieser zeichnerischen Methode war der von 1855 bis 1881 an der ETH Zürich lehrende Karl Culmann. Sein einschlägiges Werk, die Graphische Statik (erste Auflage 1866), sicherte ihm die Anerkennung der Zeitgenossen und das Andenken der Technikhistoriker. Culmann sprach auch oft von der Zeichnung als der Sprache des Ingenieurs. Für Culmann ist das Zeichnen Teil jener graphischen Methode, die nicht bloß darstellend verfährt, sondern die operativ geworden ist. Oder, in den Worten von Ekkehard Ramm: „Graphische Statik heißt, daß mechanische Größen wie Kräfte oder Verschiebungen mit rein graphischen, also geometrischen Methoden bestimmt werden, sie also nicht 
nur zur Visualisierung analytischer Zusammenhänge dient." 4

Culmanns Graphische Statik stützt sich wesentlich auf die Weiterentwicklung der Darstellenden Geometrie zur Projektiven Geometrie durch Jean Victor Poncelet. Ivor Grattan-Guinness nennt Poncelet einen „engineer educator" mit einem „mathematical hobby of projective geometry“. 5 Dieses Hobby pflegte er 1813 in russischer Kriegsgefangenschaft, wo es ihm gelang mit der Annahme von „unendlich fernen Punkten und der Methode der Projektion, überraschend einfache Beweise für eine ganze Reihe geometrischer Lehrsätze zu finden“. ${ }^{6}$ Solcher unendlich ferner Punkte hatte sich schon Gérard Desargues bedient, dessen Theorem, „das ein Bündel von parallelen Geraden mit einem Bündel von konvergierenden Geraden gleichsetzt“", seinen Sinn selbst erst im Rahmen der projektiven Geometrie erhält. $^{7}$

Als im Mai 1820 ein umfangreiches Manuskript von Poncelet über projektive Geometrie in der Akademie in Paris besprochen wird, ist die Aufnahme reserviert. Der Berichterstatter Augustin-Louis Cauchy, der in der Akademie den Platz des nach Napoleons Sturz aus politischen Gründen ausgeschlossenen Monge eingenommen hatte, schätzte die Geometrie gering. Er war auch Parteigänger der von Pierre-Simon Laplace forcierten Neuausrichtung des Unterrichts der École Polytechnique an der Analysis, wodurch die Darstellende Geometrie ihre grundlegende Rolle in der Ausbildung verlor. Gleichzeitig aber wurden damit eher Mathematiker als Ingenieure ausgebildet. Poncelet schloß sich dieser akademischen Tendenz einer „Bildfeindlichkeit“, verursacht durch die „Unanschaulichkeit“ der Analysis, nicht an.

Ebenso wie Poinsot und Coriolis war Poncelet der Auffassung, daß geometrischen Methoden in der Mechanik ein eigenständiger Platz eingeräumt werden müsse und der Anschaulichkeit (zum Beispiel eines Bewegungsvorganges) für die Technikwissenschaften ein unschätzbar hoher Wert innewohnt. ${ }^{8}$

Für Culmann war Poncelet allerdings noch zu sehr der Analysis verpflichtet. So schreibt er in der Einleitung zur ersten Auflage von Die graphische Statik:

Während jedoch Poncelet nur Geometrie verwendete, wo er musste, sonst aber der Analysis den Vorzug gab, kehrten wir es um und wendeten Geometrie an, wo wir konnten, und erzielten so Resultate, die Jeden erwarten, der zum ersten Mal es unternimmt, ein noch unbebautes Feld zu bearbeiten. ${ }^{9}$ 
Es ist insbesondere das „räumliche Anschauungsvermögen“, das den Techniker befähigen soll, „mit Leichtigkeit flache Projectionen zu Körpern (zu) ergänzen" ${ }^{10}{ }^{10}$ Damit leistet der Techniker die Übersetzung von der (flachen) Konstruktionszeichnung zum (räumlichen) technischen Objekt. Dieses wiederum bevölkert im Falle einer Neukonstruktion seine Vorstellung, um in einer Serie von Zeichnungen das Licht der Welt zu erblicken.

\section{Analytisches Konstruieren}

Culmanns Kollege am eidgenössischen Polytechnikum, Franz Reuleaux, griff die graphische Statik auf und verwendete sie im Rahmen des Maschinenbaus. Franz Reuleaux, Schüler von Ferdinand Redtenbacher in Karlsruhe, begann seine Arbeiten ganz dem Feld der graphisch-geometrischen Methode verpflichtet. Noch in seiner Studienzeit hatte er mit seinem Studienkollegen C.L. Moll einen „Verein der Maschinenbaueleven“ gegründet, „deren Mitglieder Konstruktionszeichnungen anfertigten, wobei sie auch Vorlagen aus Redtenbacher-Vorlesungen verwendeten. Diese Zeichnungen legten sie

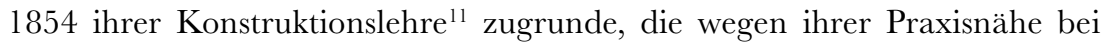
Ingenieuren und Ingenieurstudenten bald sehr beliebt war." ${ }^{\text {12 }}$ Redtenbacher erhob etwas verärgert einen Plagiatsverdacht, da er einiges aus seinen Vorlesungen wiedererkannte. Zehn Jahre später in Zürich kam Reuleaux seinem Kollegen Culmann zuvor und publizierte zwei Jahre vor dem Erscheinen der Graphischen Statik seinerseits Die graphische Statik in ihrer Anwendung auf den Maschinenbau. Schließlich nahm Reuleaux ab der dritten Auflage seines Werkes Der Constructeur die, wie er sie nun nannte, ,Graphostatik ${ }^{6}$ als Hilfslehre auf. Hier gibt es nun auch in einer Fußnote den lobenden Verweis auf Culmann und seine Graphische Statik, ,welches verdienstvolle Werk [...] überhaupt diesen Wissenszweig zuerst methodisch zusammengefaßt und in die Praxis eingeführt hat.“13 Culmann äußert sich dazu etwas abschätzig: „In Preussen wurde zunächst der Name ,graphische Statik“ in ,Graphostatik umgeändert, im übrigen aber sehr wenig geleistet. “14

Reuleaux versuchte dem Maschinenbau auf anderem Wege eine wissenschaftliche Grundlage zu geben, die ihn aus dem Gebiet der graphischen Geometrie herausführte. Die vierte Auflage von Der Construkteur enthält im Vorwort einen längeren Abriß über jene Lehre, die nun den Kern des Lebenswerkes von Reuleaux ausmachen sollte: die Kinematik oder Zwanglauflehre. Für sie entwi- 
ckelte er eine kinematische Zeichensprache, welche es nicht nur gestatten sollte, von Maschinen eine kinematische Analyse durchzuführen, indem sie die Zeichnung durch eine Formel ersetzt, sondern schließlich auch zu einer kinematischen Synthese vorzustoßen, also vorsichtig ausgedrückt, zu einer Lehre der Erfindung von neuen Maschinen zu gelangen. ${ }^{15}$

Anders als bei der Analyse von Fachwerken, für die die graphische Statik Culmanns ganz besonders geeignet war, hat man es im Maschinenbau mit einer oft verwirrenden Fülle von sinnreich kombinierten Elementen zu tun. Es ist nicht leicht, hierbei einen zeichnerischen Überblick zu bewahren und insbesondere von der Zeichnung auf die Bewegungsformen zu schließen. Dieses Problem hatte Charles Babbage zur Erfindung seiner Mechanical Notation geführt, die zusammen mit jener von Robert Willis als Vorbild für Reuleaux's kinematische Zeichensprache anzusehen ist. ${ }^{16}$

Babbage, dem die Erfindung der Mechanical Notation überaus wichtig war, charakterisiert sie als „System sehr einfacher Zeichen“, mit welchem sich die komplizierteste Maschine „fast ganz ohne die Hilfe von Wörtern erklären ließ ${ }^{\prime 1}{ }^{17}$ Die Zeichnung der Maschine bzw. der Teile der Maschine war allerdings unumgänglich, die Notation wurde auf ihr notiert und im Zusammenklang gelesen. Damit beanspruchte Babbage nicht nur die konkrete Form und relative Position aller Maschinenteile angeben zu können, sondern auch ihren zeitlichen Bewegungsablauf. Aber schon bei Babbage war mit dieser Notation mehr verbunden, als das praktische Verständnis von Maschinen. Er erhebt einen gewissen wissenschaftlichen Anspruch:

Durch ihre Anwendung auf geometrische Zeichnungen gibt sie uns eine neue exakte Wissenschaft [demonstrative science] an die Hand, die zu beweisen erlaubt, ob es eine bestimmte Maschine wirklich geben kann oder nicht, und, wenn es sie geben kann, ob sie auch zu dem gewünschten Ziel führen wird. ${ }^{18}$

Reuleaux entwickelt seinen Begriff der Kinematik in Abgrenzung der Maschinenwissenschaft von der allgemeinen Mechanik und damit der Ablösung von der Physik. ${ }^{19}$ Während die Natur von sensiblen Kräften beherrscht ist, wirken in der Maschinenkonstruktion latente. Während also z. B. die Kreisbahn eines Planeten durch störende Kräfte beeinflußt werden kann, gilt es bei der Maschine diese verläßlich auszuschalten. Damit gelangt Reuleaux zu seiner grundlegenden Definition der Maschine: „Eine Maschine ist eine Verbindung widerstandsfähiger Körper, welche so eingerichtet ist, dass mittelst 
ihrer mechanische Naturkräfte genöthigt werden können, unter bestimmten Bewegungen zu wirken. ${ }^{\text {"20 }}$ Es geht also darum bestimmte Bewegungsformen zu erzwingen, daher der Name Zwanglauflehre für die zugrundeliegende Wissenschaft Kinematik. Diese ist für Reuleaux der grundlegende Teil der Maschinenwissenschaft, weil ihm die Bewegungserzwingung das allgemeine Prinzip der Maschine ist.

Die Elemente der Maschine sind nun nicht mehr in klassischer Weise Rad, Rolle, schiefe Ebene, Hebel und dergleichen mehr, sondern Elementenpaare, wie sie etwa Schraube und Mutter darstellen. Aus solchen Elementenpaaren lassen sich kinematische Ketten aufbauen, welche, wenn sie die Eigenschaft besitzen, „daß jedem Glied nur eine Relativbewegung gegenüber jedem anderen Glied möglich ist" zwangläufig geschlossene Kette heißt. Wird ein Glied der Kette festgestellt und bildet sonach eine Art „absolutes Bezugssystem“ für alle anderen Kettenglieder, dann spricht Reuleaux von Mechanismus oder Getriebe. Wenn darauf eine Kraft einwirkt, dann ist das Ganze eine Maschine. Damit fügt Reuleaux in die Maschinenlehre eine Getriebelehre ein, die ungeachtet aller anderen Ansprüche, die Reuleaux damit verbunden hat, zum bleibenden Bestand der Maschinenbaulehre gezählt wurde. Seine dafür entwickelte kinematische Zeichensprache allerdings konnte sich nicht durchsetzen.

Schließlich formuliert Reuleaux sein wissenschaftliches Credo:

Es handelt sich darum, die Maschinenwissenschaft der Deduktion zu gewinnen. Es handelt sich darum, deren Lehrgebäude so zu gestalten, dass es sich auf wenigen, ihm eigenthümlichen Grundwahrheiten erhebt. Auf deren Ernst und Einfachheit muss der ganze Gestaltenreichthum zurückführbar sein, aus ihnen muss er umgekehrt entwickelbar sein. ${ }^{21}$

\section{Die Gegenbewegung der Praktiker ${ }^{22}$}

Gegen dieses Credo hat sich ausdrücklich Alois Riedler gewandt, ein Kollege von Reuleaux an der Technischen Hochschule in Berlin-Charlottenburg seit 1888. In einer Schrift von 1899, also schon nach Reuleaux's Abgang von der TH-Charlottenburg (1895), setzt sich Riedler mit dem Wissenschaftsanspruch Reuleaux's auseinander. Er polemisiert dabei gegen das deduktive Verfahren, gegen die Kategorie des Gesetzes, die nunmehr von anderen Grundsätzen überholt sei: „Nur das wirkliche Verhalten der Konstruktionsmaterialien ist 
massgebend, nur die wirklichen Vorgänge gegenüber den vielfältigen, gleichzeitig auftretenden Ursachen sind entscheidend." ${ }^{23}$ Der empirische Zug, den die Technikwissenschaften angenommen haben, läßt nicht mehr nur als wissenschaftlich gelten, ,was sich bequem mathematisch oder in einfacher Deduktion aus Annahmen ableiten lässt. “24

Als Nachklang ziemlich polemisch getönter Auseinandersetzungen setzt Riedler zu einer erklärenden Verteidigung an, die gleichzeitig eine Kritik am alten Unterricht im technischen Zeichnen ist:

Im Konstruktionsunterricht, den ich als >Vorbild auch entwerthet haben soll, fand ich beim Antritt meiner Thätigkeit an der Berliner Hochschule ein >Maschinenzeichnen vor, in welchem romanische Kapitäle, Stopfbüchsen und Schubstangen unmöglicher Bauart nach Vorlagen, >Vorbildern< schlechtester Art, abgezeichnet wurden; die Zeichnungen wurden dann mühsam mit Schlagschatten und Komplementärfarben abgetuscht. In den Uebungen in den >Maschinenelementen $>$ Konstrukteur , den jeder Studirende neben sich beim Reissbrett haben musste, und nach seinen >Gesetzen Maschinentheile `berechnet<, d. h. auf Grund einer Annahme, z. B. einer einzigen statischen Kraftwirkung, die einzelnen Abmessungen ermittelt. Die so entstandenen Maschinentheile waren allerdings nicht ausführbar und entsprachen keinem praktischen Zwecke; ebensowenig aber den vielfältigen wissenschaftlichen Forderungen. ${ }^{25}$

Schließlich ist daran zu erinnern, daß Riedler ein Werk verfaßt hat, mit dem er zum Begründer des modernen technischen Zeichnens wurde und das er im Vorwort zur zweiten Auflage ausdrücklich als Kampfschrift bezeichnet: Das Maschinen-Zeichnen. ${ }^{26}$ Damit sei auf die hohe Wertschätzung verwiesen, die Riedler dem Zeichnen als Element der Ausbildung und der Praxis des Ingenieurs entgegenbrachte. Viele seiner eher kleineren Schriften sind der Ingenieurausbildung gewidmet, in denen immer wieder kehrende Themen abgehandelt werden, die um den Begriff des räumlichen Vorstellungsvermögens kreisen. Dieses auszubilden, muß der Eckpfeiler jeder Ingenieurausbildung sein und dazu eignet sich die Darstellende Geometrie in hervorragender Weise. So betont er 1895:

Die Kennzeichnung ist äußerst einfach: ein entwickeltes Vorstellungsvermögen ist die Grundlage jeder schaffenden Thätigkeit, und Ingenieurthätigkeit 
ist durchaus möglich ohne nennenswerthe mathematische Bildung [...] Ohne bildendes, gestaltendes und wiedergebendes Vorstellungsvermögen hingegen ist keine schaffende Ingenieurthätigkeit möglich. Die Ausbildung dieses Vermögens ist die wichtigsten Grundlage der Ingenieur-Erziehung; das wichtigste Hülfsmittel hierzu ist der geometrische Unterricht. ${ }^{27}$

In seinem erwähnten Buch über das Maschinen-Zeichnen hebt Riedler den Charakter der Zeichnung als Sprache hervor:

Die Zeichnung ist insbesondere die Sprache des Ingenieurs, eine ausdrucksvolle internationale Sprache, die aber stets, im Gegensatz zu allen anderen, erschöpfend, klar und eindeutig, jedes Mißverständnis ausschließend gesprochen werden muß. ${ }^{28}$

Was diese Sprache erfüllen muß und welche Form sie daher anzunehmen hat, läßt sich aus einem Manuskript von Riedler entnehmen.

Die Sprache der Zeichnung ist durch die Technik überhaupt höher entwickelt, als es je einer Lautsprache gelingen könnte. Sie ist die einzige völlig klare und eindeutige und dabei ausdrucksvolle Sprache, frei von Deutungen und Missverständnissen [...] Die Werkzeichnung einer technischen Gestaltung ist das Ausdrucksmittel, wodurch der Gestalter seine Gedanken und Absichten den ausführenden Arbeitern so mitteilt, dass diese die Gestaltung in zweckrichtiger Form verwirklichen, ohne dass irgend eine mündliche Anweisung notwendig wird. Eine vollkommene Sprache! ${ }^{29}$

Die Werkzeichnung als stummer Befehl an die Werkstätte, eine technische Konstruktion genau so auszuführen, wie es die Zeichnung vorschreibt, das erinnert in der Tat an eine Sprache, wie sie Wittgenstein beschrieben hat:

Man kann sich denken, daß ein Mensch die Sprache erfindet; daß er die Erfindung macht, andere menschliche Wesen statt seiner arbeiten zu lassen indem er sie durch Strafe und Belohnung abrichtet, auf Zurufe hin gewisse Tätigkeiten zu verrichten. Diese Erfindung wäre analog der Erfindung einer Maschine. ${ }^{30}$

Was kann man sich vorstellen unter Erfindung einer Maschine? Erfinden heißt etwas in die Welt setzen wollen, was es in ihr noch nicht gibt. Man hat 
davon kein Bild vor Augen, weil man dieses Bild erst herstellen muß. Eugene Ferguson hat nun dem Ingenieur ein besonderes Auge angedichtet, das innere Auge, the mind's eye, mit dem der erfindende Ingenieur der Neukonstruktion zuerst ansichtig wird. ${ }^{31}$ Ferguson sagt wenig zum Charakter dieser Maschinenansicht. Man kann annehmen, daß dieses geistige Bild eine vage Gestalt hat, es wird ja gleichsam halluzinatorisch hervorgerufen, wobei auch schon gewisse Vorstellungen über den materiellen Charakter eingehen, über wirkende Kräfte und die dafür notwendige Dimensionierung etc. Ferguson berichtet von Nicola Tesla, daß dieser Maschinen im Kopf konstruiert habe und sie dort probelaufen ließ um nach einiger Zeit nachzusehen ob sie noch funktionieren. Weniger begabte Ingenieure werden eine erste Skizze der Maschine anfertigen, die man gerne ,sprechende Skizze' nennt, weil sie eingebettet ist in einen umrahmenden erklärenden Diskurs und der Diskussion dient. Diese Skizze bzw. die Serie von Skizzen, beschränkt sich auf das entscheidend Neue, und ohne den Regeln der Darstellenden Geometrie zeichnerisch zu folgen, setzt sie doch das durch sie eingeübte räumliche Vorstellungsvermögen voraus. Hat sich die Erfindung hier bewährt, kann man den Prozess der Konstruktion mit der Berechnung der Maschine einleiten. Hier kann man an die graphische Statik erinnern, die als graphisches Rechnen ein Analogrechnen war, wie es im Zeitalter vor dem Computer für Ingenieure gang und gäbe war mit dem vornehmsten Recheninstrument, dem Rechenschieber, dem Culmann auch ein Kapitel seiner Graphischen Statik widmet. Hat man die Maschine durchgerechnet, dann wird man an die Erstellung der Serie von Werkstattzeichnungen gehen, die als Vorschriften zu gelten haben. Hier geht die Zeichnung eben in die Sphäre des Befehls ein, eines Befehls, der im normalen Fall keine weiteren Erklärungen mehr nötig hat, sondern alle notwendige Information für die Herstellung der wirklichen Maschine enthält. Die Eineindeutigkeit der Werkstattzeichnung betrifft vor allem ihre Bemaßung. Mit diesen Zeichnungen (und der beigefügten Stückliste) endet die Arbeit des Ingenieurs, der immer nur Papiermaschinen herstellt. In diesen Papiermaschinen erschöpft sich schließlich die Tätigkeit des Ingenieurs. 


\section{Anmerkungen}

1 Kelly Hamilton, „Wittgenstein and the Mind's Eye“, in Wittgenstein. Biography and Philosophy edited by James C. Klagge (Cambridge: Cambridge University Press 2001), 53-97; Michael A. R. Biggs, Visualisation and Wittgenstein's ,Tractatus (http://wab.aksis.uib.no/sept1914/graphics/crc_biggs.pdf).

2 Brian McGuiness, Wittgensteins frühe Fahre (Frankfurt: Suhrkamp 1988), 110.

3 Das betont Harold Belofsky, „Engineering Drawing - a Universal Language in Two Dialects“, Technology and Culture 32 (1991), 23-46, $26 \mathrm{f} .:$,... there can be little doubt that third angle is easier to master and probably results in fewer drawing errors with drafters of equal ability." Die Steinschneider in den Werkstätten von Mézières, wo Monge an der Militärschule unterrichtete, zeichneten ihre groben Schnittlinien auf die Oberflächen der Steinkuben im dritten Quadranten.

4 Ekkehard Ramm, „Vorwort“, in Bertram Maurer, Karl Culmann und die graphische Statik (Berlin: Verlag für Geschichte der Naturwissenschaft und Technik 1998), xiii.

5 Ivor Grattan-Guinness, Convolutions in French Mathematics, 1800-1840, Volume II: The Turns (Basel, Boston, Berlin: Birkhäuser 1990), 1052.

6 Maurer, Culmann,109.

7 Hubert Damisch, Der Ursprung der Perspektive (Zürich: diaphanes 2010), 67.

8 Andreas Kahlow, ,Jean Victor Poncelet und die Schwierigkeiten des visuellen Denkens in den klassischen Technikwissenschaften“, Dresdener Beiträge zur Geschichte der Technikwissenschaften 23/1 (1994) 70-78, hier 72.

9 Karl Culmann, Die graphische Statik (Zürich: Meyer \& Zeller 1866), XII.

10 Culmann: Statik, VIII.

111854 unter dem Titel Constructionslehre für den Maschinenbau erschienen.

12 H.-J. Braun und W. Weber, „Ingenieurwissenschaft und Gesellschaftspolitik. Das Wirken von F. Reuleaux, " in Wissenschaft und Gesellschaft. Beiträge zur Geschichte der Technischen Universität Berlin 1879-1979, herausgegeben von R. Rürup, 1. Band (Berlin: Springer 1979), 285-300, hier 286.

13 Franz Reuleaux, Der Konstrukteur. Ein Handbuch zum Gebrauch beim MaschinenEntwerfen, 4. Auflage (Braunschweig: Vieweg und Sohn 1899), 76.

14 Karl Culmann, Die graphische Statik, 2. Aufl., 1. Band (Zürich: Meyer \& Zeller 1875), IX.

15 Reuleaux, Der Konstrukteur, XXIV: „Man könnte also die kinematische Synthese als eine Theorie des Maschinenerfindens ansehen. Das kann sie sein, aber nur in einem eingeschränkten Sinne. [...] Man kann zunächst gewisse erfinderische Auf- 
gaben, die schon gelöst sind, als auf synthetischem Wege lösbar nachweisen, und kann danach zu anderen, noch ungelösten Aufgaben mindestens Wege der Lösung andeuten."

16 Vgl. dazu Francis C. Moon, „Robert Willis and Franz Reuleaux: Pioneers in the Theory of Machines“, Notes and Records of the Royal Society London 57 (2), (2003), 209-230.

17 Charles Babbage, „Über die Mechanical Notation (1864),“ in Babbages RechenAutomate, herausgegeben von Bernhard Dotzler (Wien: Springer 1996), 201-204, hier 201.

18 Babbage, „Notation“, 202.

$19 \mathrm{Vgl}$. für das folgende die grundlegende Arbeit von Karl-Norbert Ihmig, „Das Verhältnis von Mathematik und Kinematik bei Franz Reuleaux", in Mathematik und Technik im 19.Jahrhundert in Deutschland. Soziale Auseinandersetzung und philosophische Problematik, herausgegeben von Susann Hensel, Karl-Norbert Ihmig und Michael Otte (Göttingen: Vandenhoeck \& Ruprecht 1989), 112-148.

20 Franz Reuleaux, Theoretische Kinematik, (Braunschweig: Vieweg und Sohn 1875), 38.

21 Reuleaux, Theoretische Kinematik, 26.

22 In diese Gegenbewegung ordnet Wolfgang König, Künstler und Strichezieher. Konstruktions- und Technikkulturen im deutschen, britischen, amerikanischen und französischen Maschinenbau zwischen 1850 und 1930 (Frankfurt: Suhrkamp 1999) 55, Alois Riedler ein.

23 Alois Riedler, Die Stellung des Herrn Reuleaux zu den technischen Wissenschaften (Berlin 1899), 3.

24 Riedler, Reuleaux, 3.

25 Riedler, Reuleaux, 3.

26 Alois Riedler, Das Maschinen-Zeichnen. Begründung und Veranschaulichung der sachlich notwendigen zeichnerischen Darstellungen und ihres Zusammenhanges mit der praktischen Ausführung, 2. Auflage, (Berlin: Springer 1913), IV.

27 Alois Riedler, Zur Frage der Ingenieur-Erziehung (Berlin 1895), 19.

28 Riedler, Maschinen-Zeichnen, 2.

29 Alois Riedler, Form und Zielbegrenzung (maschinenschriftliches Manuskript datiert Mai 1928), Nachlaß Alois Riedler, Technisches Museum Wien, Riedler-NL BPA $3645,6 \mathrm{f}$.

30 Ludwig Wittgenstein, Philosophische Grammatik (Frankfurt: Suhrkamp 1973), 187.

31 Eugene S. Ferguson, Das innere Auge. Von der Kunst des Ingenieurs (Basel: Birkhäuser 1993). 
